\title{
Mitigating airborne transmission of SARS-CoV-2
}

\author{
Sarah Addleman MDCM, Victor Leung MD, Leyla Asadi MD MPH, Abdu Sharkawy MD, Jennifer McDonald MD
}

Cite as: CMAJ 2021 July 5;193:E1010-1. doi: 10.1503/cmaj.210830; early-released June 8, 2021

A $t$ the outset of the COVID-19 pandemic, understanding of respiratory virus transmission was founded on a conceptual framework that dichotomized infectious particles into droplets that are $>5 \mu \mathrm{m}$ and "drop" close to the source, versus aerosols that are $<5 \mu \mathrm{m}$ and remain suspended in air. However, aerosol scientists challenged this traditional categorization: particles as large as $100 \mu \mathrm{m}$ can remain suspended in air and be inhaled. ${ }^{1}$ In November 2020, the Public Health Agency of Canada (PHAC) recognized that SARS-CoV-2 can be transmitted by aerosol, and more recent updates from the World Health Organization $(\mathrm{WHO})^{2}$ and Centers for Disease Control and Prevention (CDC) ${ }^{3}$ highlight both short- and long-range aerosol transmission. Despite this, public health guidance in Canada has not been adequately updated to address airborne transmission, possibly because of ongoing debate over its relative importance compared with other modes of transmission. We argue that it is not necessary to reach consensus on the predominance of airborne transmission before making meaningful changes to Canada's public health guidance. We call for updated messaging and policies on ventilation and masks - informed by multidisciplinary collaboration - to ensure the highest standards of safety for Canada's communities.

The CDC states that SARS-CoV-2 spreads by exposure to infectious respiratory fluids through aerosol inhalation, deposition of droplets (splashes and sprays), and touch, and that aerosol inhalation, although more likely at close range because aerosols are more concentrated at their source, also occurs at longer distances, depending on environmental conditions. ${ }^{3}$ Airborne transmission of SARS-CoV-2 is supported by its asymptomatic transmission, superspreading events, long-range transmission confirmed by contact tracing and genomic sequencing, nosocomial infections despite strict adherence to contact and droplet personal protective equipment (PPE), and animal studies showing transmission via an air duct. ${ }^{4}$ Although air sampling of live pathogens in nonlaboratory settings is challenging - researchers have been unsuccessful with measles and tuberculosis, both diseases with airborne transmission - viable SARS-CoV-2 has been sampled from the air in a hospital room and a car driven for 15 minutes by a mildly symptomatic patient. ${ }^{4}$ Tuberculosis also infects primarily those in close proximity despite having long-range transmission potential.

Having established that SARS-CoV-2 can infect at both short and long range by aerosol inhalation, use of the term "airborne" accurately reflects transmission through the air and permits clear communication with the public. The importance of ventilation

\section{KEY POINTS}

- Short- and long-range aerosol inhalation, or airborne transmission, of SARS-CoV-2 has been recognized by international public health agencies.

- Canadian public health guidance and policies should be updated to address this mode of transmission.

- Ventilation is a key mitigation measure against airborne transmission, and recommendations and funding should be provided to business and schools for assessments and upgrades.

- Guidelines for personal protective equipment in health care settings and essential workplaces should be revisited, with the goal of implementing more widespread use of N95 respirators.

- Addressing airborne transmission requires the expertise of interdisciplinary teams to inform solutions that can end this pandemic faster.

and filtration, wearing masks indoors even when distanced, and choosing outdoor spaces for gathering becomes more intuitive when airborne transmission is explicitly acknowledged.

Ventilation is a key element in the mitigation against longrange aerosol transmission, and actionable advice and funding to prioritize ventilation assessments and upgrades should be widely disseminated to businesses and schools. Unproven mitigation measures like "deep cleaning" and plexiglass installations as barriers to droplet spread persist as misplaced investments that can provide a false sense of security. The risk of fomite transmission is estimated to be low and plexiglass can actually impede air flow, potentially leading to increased aerosol transmission. ${ }^{5}$ The CDC has published a toolkit of interventions to improve ventilation in buildings, many of which are easily achievable at relatively low cost. ${ }^{6}$ Health Canada recently updated its guidelines for assessing and improving residential indoor air quality using carbon dioxide levels ${ }^{7}-$ an easily measured proxy for ventilation. Similar guidelines are urgently needed for other indoor spaces, in particular our schools; $;$ in addition to protecting against airborne transmission, there is a growing body of literature on the general health and cognitive benefits of improved ventilation.

Health care staff involved in prolonged, close-proximity patient care have been shown to be at particularly high risk of acquiring SARS-CoV-2 infection, despite contact and droplet PPE. ${ }^{9}$ Large outbreaks in long-term care homes have been a perfect storm: multiple 
residents in the highly contagious presymptomatic and early symptomatic phases of illness, living in settings with ventilation standards below those of modern hospitals. These outbreaks show that applying only droplet- and contact-control models to older health care institutions can have devastating results. High attack rates in long-term care facilities are unlikely to be adequately explained by PPE doffing breaches, and more focus on mask quality and ventilation (including in break rooms) would be prudent.

Although good ventilation can mitigate long-range aerosol transmission of SARS-CoV-2, the WHO and CDC's acknowledgement of airborne spread at close range highlights an urgent need to revisit PPE guidelines in all Canadian health care settings. Highquality masks such as N95 respirators are currently required only for "aerosol-generating medical procedures." This guidance recognizes that $\mathrm{N} 95$ respirators are the gold standard for protection against short-range aerosol inhalation. Although surgical masks do offer source control and some aerosol protection, ${ }^{10}$ their efficacy against inhaled aerosols is highly variable. Observational studies have found higher rates of SARS-CoV-2 infection among health care workers using surgical masks than among those using N95 respirators, ${ }^{9}$ and the CDC recommends N95s, along with gown, gloves and eye protection, when caring for patients with suspected or confirmed SARS-CoV-2 infection. ${ }^{11}$ Recent studies have shown that exertional activities and coughing produce more aerosols than some aerosol-generating medical procedures. ${ }^{12}$ Canadian health care workers in all settings should have consistent and reliable access to N95 respirators, and be informed that these are not reserved only for aerosol-generating procedures; this suggestion aligns with a recent $\mathrm{PHAC}$ communication. ${ }^{13}$

Outside of the health care setting, essential workers in closeproximity settings such as meat-processing plants, manufacturing and distribution centres have been disproportionately affected by COVID-19. Although social measures such as paid sick leave are vital, standardized masks (ideally, fit-tested respirators) are still a critically important protective measure. Concerns about N95 shortages have diminished and there is increased local production, including of comfortable models with a more universal fit. Furthermore, ill-conceived regulations that permit removal of masks indoors if able to maintain a $2 \mathrm{~m}$ distance do not account for transmission in shared room air, and continue to put essential workers and the public at risk.

We have Canadian expertise in engineering and occupational hygiene that can help inform policies regarding ventilation and masks. Although highly efficacious vaccines are heralding an end to the COVID-19 pandemic in Canada, multidisciplinary collaboration can help end it faster. It took decades for the medical community to accept that tuberculosis and measles were airborne diseases. The science on airborne transmission of SARS-CoV-2 has, in contrast, moved fast. It is time for Canadian guidance and policies to follow swiftly too.

\section{References}

1. Tang JW, Bahnfleth WP, Bluyssen PM, et al. Dismantling myths on the airborne transmission of severe acute respiratory syndrome coronavirus (SARS-CoV-2). J Hosp Infect. 2021;110:89-96.

2. Coronavirus disease (COVID-19): How is it transmitted? [news release]. Geneva: World Health Organization; modified 2021 Apr. 30. Available: https:// www.who.int/news-room/q-a-detail/coronavirus-disease-covid-19-how-is-it -transmitted (accessed 2021 May 2).
3. Scientific brief: SARS-CoV-2 Transmission. Atlanta: Centers for Disease Control; updated 2021 May 7. Available: https://www.cdc.gov/coronavirus/2019-ncov/ science/science-briefs/sars-cov-2-transmission.html (accessed May 22 2021).

4. Greenhalgh T, Jimenez JL, Prather KA, et al. Ten scientific reasons in support of airborne transmission of SARS-CoV-2. Lancet 2021;397:1603-5.

5. Lessler J, Grabowski MK, Grantz KH, et al. Household COVID-19 risk and inperson schooling. Science $2021 \mathrm{Apr}$. 29 [Epub ahead of print]. doi: 10.1126/ science.abh2939.

6. Ventilation in buildings. Atlanta: Centers for Disease Control; modified 2021 Mar. 23. Available: https://www.cdc.gov/coronavirus/2019-ncov/community/ ventilation.html\#considerations (accessed 2021 May 22).

7. Recommended long-term exposure limit. In: Residential Indoor Air Quality Guidelines: Carbon Dioxide. Ottawa: Health Canada; modified 2021 Apr. 23. Available: https://www.canada.ca/en/health-canada/services/publications/ healthy-living/residential-indoor-air-quality-guidelines-carbon-dioxide.html\#sec71 (accessed 2021 May 22).

8. Gettings J, Czarnik M, Morris E, et al. Mask use and ventilation improvements to reduce COVID-19 incidence in elementary schools - Georgia, November 16December 11, 2020. MMWR Morb Mortal Wkly Rep 2021;70:779-84.

9. Chou R, Dana T, Buckley DI, et al. Epidemiology of and risk factors for coronavirus infection in health care workers: a living rapid review. Ann Intern Med 2020;173:120-36.

10. Cheng Y, Ma N, Witt C, et al. Face masks effectively limit the probability of SARS-CoV-2 transmission. Science 2021 May 20 [Epub ahead of print]. doi: $10.1126 /$ science.abg6296.

11. Interim infection prevention and control recommendations for healthcare personnel during the coronavirus disease 2019 (COVID-19) pandemic. Atlanta: Centers for Disease Control; modified 2021 Feb. 23. Available: https://www. cdc.gov/coronavirus/2019-ncov/hcp/infection-control-recommendations.html (accessed 2021 May 22).

12. Hamilton F, Arnold D, Bzdek BR, et al. Aerosol generating procedures: Are they of relevance for transmission of SARS-CoV-2? Lancet Respir Med 2021 May 6 [Epub ahead of print]. doi: 10.1016/S2213-2600(21)00216-2.

13. Njoo H. Response from the Public Health Agency of Canada [letter]. Ottawa: Public Health Agency of Canada; 2021 Mar. 24. Available: https://www.wigmorising. ca/wp-content/uploads/2021/04/Response-from-the-Public-Health-Agency-of -Canada.pdf (accessed 2021 May 2).

Competing interests: Leyla Asadi reports receiving a grant from the Alberta Health Innovates Clinician Fellowship. No other competing interests were declared.

This article has been peer reviewed.

Affiliations: Department of Emergency Medicine (Addleman), University of Ottawa, Ottawa, Ont.; Division of Medical Microbiology (Leung), Department of Pathology and Laboratory Medicine, and Division of Infectious Diseases (Leung), Department of Medicine, University of British Columbia, Vancouver, BC; Department of Medicine (Asadi), University of Alberta, Edmonton, Alta.; Division of Infectious Diseases (Sharkawy), Department of Medicine, University of Toronto, Toronto, Ont.; Division of Physical Medicine and Rehabilitation (McDonald), Department of Medicine, University of Ottawa, Ottawa, Ont.

Contributors: Sarah Addleman and Jennifer McDonald contributed to the conception and design of the work and drafted the manuscript. All of the authors revised it critically for important intellectual content, gave final approval of the version to be published and agreed to be accountable for all aspects of the work.

Content licence: This is an Open Access article distributed in accordance with the terms of the Creative Commons Attribution (CC BY-NCND 4.0) licence, which permits use, distribution and reproduction in any medium, provided that the original publication is properly cited, the use is noncommercial (i.e., research or educational use), and no modifications or adaptations are made. See: https://creativecommons.org/ licenses/by-nc-nd/4.0/

Correspondence to: Sarah Addleman, saddlemanfrankel@toh.ca 